\title{
Annular Bragg resonators (ABR): the ideal tool for biochemical sensing, nonlinear optics, and cavity QED
}

Jacob Scheuer, William M. J. Green, Amnon Yariv

Jacob Scheuer, William M. J. Green, Amnon Yariv, "Annular Bragg resonators (ABR): the ideal tool for biochemical sensing, nonlinear optics, and cavity QED," Proc. SPIE 6123, Integrated Optics: Devices, Materials, and Technologies X, 61230S (24 February 2006); doi: 10.1117/12.640285

Event: Integrated Optoelectronic Devices 2006, 2006, San Jose, California, United States 


\title{
Annular Bragg Resonators (ABR) - The Ideal Tool for Biochemical Sensing, Nonlinear Optics and Cavity QED
}

\author{
Jacob Scheuer*, William M. J. Green and Amnon Yariv \\ Departments of Applied Physics and Electrical Engineering, California Inst. of Technology, 1200 E. \\ California Blvd., Pasadena, CA USA 91125
}

\begin{abstract}
Circular resonators are fundamentally interesting elements that are essential for research involving highly confined fields and strong photon-atom interactions such as cavity QED, as well as for practical applications in optical communication systems as and biochemical sensing. The important characteristics of a ring resonator are the Q-factor, the free spectral range (FSR) and the modal volume, where the last two are primarily determined by the resonator dimensions. The TotalInternal-Reflection (TIR) mechanism employed in "conventional" resonators couples between these characteristics and limits the ability to realize compact devices with large FSR, small modal volume and high Q.

Recently, we proposed and analyzed a new class of a resonator in an annular geometry that is based on a single defect surrounded by radial Bragg reflectors on both sides. The radial Bragg confinement breaks the link between the characteristics of the mode and paves a new way for the realization of compact and low loss resonators. Such properties as well as the unique mode profile of the ABRs make this class of devices an excellent tool for ultra-sensitive biochemical detection as well as for studies in nonlinear optics and cavity QED.

Keywords: Bragg, resonators, distributed feedback, laser, sensing, nonlinear optics.
\end{abstract}

\section{INTRODUCTION}

Circular resonators are key ingredients in the realization of many basic components needed for advanced optical communication systems. During the last decade, numerous circular-resonator based applications such as lasers, filters, add/drop multiplexers, ${ }^{2}$ modulators ${ }^{3}$ and delay lines ${ }^{4}$ have been suggested and demonstrated. In addition, the applicability of circular resonators was shown to extend beyond telecommunication to the fields of sensing, ${ }^{5}$ spectroscopy and standardization, ${ }^{6}$ as well as to basic research in QED, nonlinear optics and other related fields. ${ }^{7}$

For many of these applications, the circular resonator is required to exhibit low losses or, equivalently, a high quality factor $(Q)$. In addition to high $Q$, it often desirable that the resonator has small dimensions or, equivalently, exhibits large free spectral range (FSR). Unfortunately, for conventional resonators, which utilize total internal reflection (TIR) as the radial confinement mechanism, these requirements are contradicting. To exhibit large FSR, a circular resonator is required to have short circumference and small bending radius. In these conditions, the efficiency of the TIR confinement mechanism is significantly impaired, leading to larger power dissipation and lower $Q$-factor. ${ }^{8}$

Recently, we have suggested utilizing Bragg reflection instead of TIR as the radial confinement mechanism in order to break the link between the FSR and the loss and facilitate low-loss, large FSR circular resonators. ${ }^{911}$ This concept is illustrated in Fig 1. A disk [Fig. 1(A)] or a circumferentially guiding defect [Fig. 1(B)] is located within a medium which consists of annular Bragg layers. The confinement of the modal field within the defect [Fig. 1(B)] or in the disk [Fig. $1(\mathrm{~A})$ ] is accomplished by Bragg reflection instead of TIR. Unlike conventional resonators, the reflectivity of the Bragg mirror can be increased simply by adding more layers. As a result, the radius of the defect (or the disk) can be reduced almost arbitrarily without the penalty of higher bending losses.

* koby@ caltech.edu; Phone: 1626 395-4413; Fax: 1626 405-0928; www.its.caltech.edu/ koby/

Integrated Optics: Devices, Materials, and Technologies X, edited by Yakov Sidorin, Christoph A. Waechter, Proc. of SPIE Vol. 6123, 61230S, (2006) · 0277-786X/06/\$15 · doi: 10.1117/12.640285 
In this paper we study the characteristics of these new types of circular resonators with an emphasis on their advantages for applications in optical sensing, nonlinear processing and cavity quantum electrodynamics (QED).

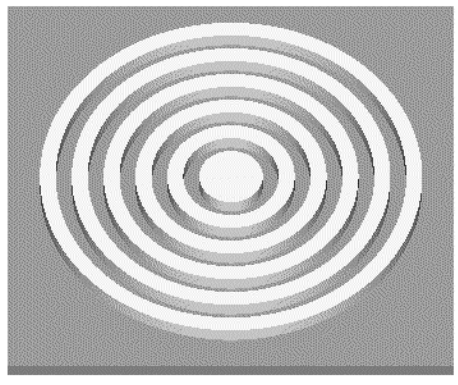

(A)

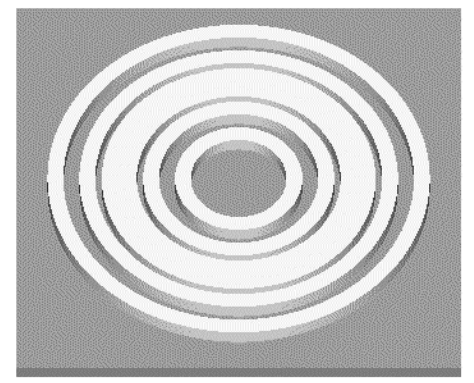

(B)

Figure 1. An illustration of A) a Bragg disk resonator; B) an annular Bragg resonator structure.

\section{BASIC THEORY}

We consider an azimuthally symmetric structure as illustrated in Figure 2. The guiding defect, which is comprised of a material of refractive index $n_{\text {defect, }}$ is surrounded by distributed Bragg reflectors on both sides, where the reflectors' layers are of refractive indices $n_{1}$ and $n_{2}$. All the electromagnetic field components can be expressed in terms of the $z$ component of the electric and magnetic fields. ${ }^{12}$ These satisfy the Helmholtz equation which in cylindrical coordinates is given by:

$$
\left[\rho^{2} \frac{\partial^{2}}{\partial \rho^{2}}+\rho \frac{\partial}{\partial \rho}+\left(k^{2}(\rho)-\beta^{2}\right) \rho^{2}-m^{2}\right]\left(\begin{array}{l}
E_{z} \\
H_{z}
\end{array}\right)=0
$$

where $\rho$ and $\theta$ are the radial and azimuthal coordinates respectively, $m$ is the azimuthal wavenumber, $\beta$ is the $z$ component propagation coefficient, $k(\rho)=k_{0} n(\rho), k_{0}$ is the wavenumber in vacuum, and the refractive index $n(\rho)$ equals either $n_{\text {defect }}, n_{1}$, or $n_{2}$ according to the radius $\rho$.

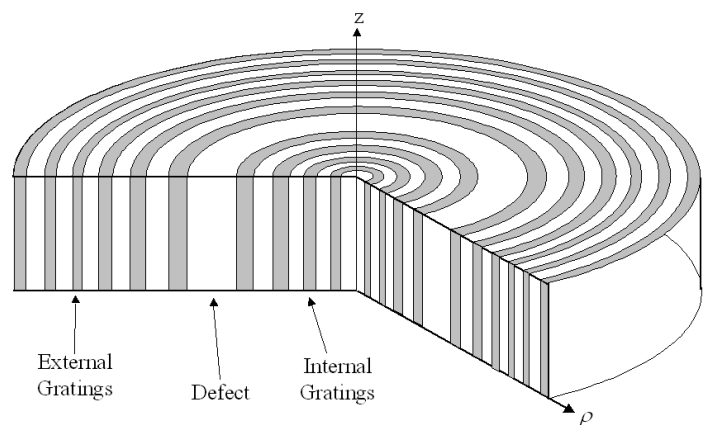

Figure 2. An illustration of the annular defect mode resonator structure.

The general solution of equation (1) can be expressed by a superposition of the Bessel functions of the first and second kind.

$$
\begin{aligned}
& E_{z}=\left[A \cdot J_{m}\left(\sqrt{k_{j}^{2}-\beta^{2}} \rho\right)+B \cdot Y_{m}\left(\sqrt{k_{j}^{2}-\beta^{2}} \rho\right)\right] \cdot \cos (\beta \cdot z+\varphi) \cdot \exp (i m \theta) \\
& H_{z}=\left[C \cdot J_{m}\left(\sqrt{k_{j}^{2}-\beta^{2}} \rho\right)+D \cdot Y_{m}\left(\sqrt{k_{j}^{2}-\beta^{2}} \rho\right)\right] \cdot \sin (\beta \cdot z+\varphi) \cdot \exp (\operatorname{im} \theta)
\end{aligned}
$$

where $k_{j}$ is the material wavenumber in the $j^{\text {th }}$ layer. 
The other field components can be easily derived from $E_{z}$ and $H_{z}{ }^{12}$. Introducing (2) into (3) yields all the field components in the $j^{\text {th }}$ layer. The parallel component of the fields $-E_{z}, H_{z}, E_{\theta}, H_{\theta}$ must be continuous at the interfaces separating successive layers. This requirement can be written in from of a transfer matrix, connecting the amplitude vector $[A B C D]$ in the $j^{\text {th }}$ and $j+1$ layers:

$$
\left[\begin{array}{llll}
A & B & C & D
\end{array}\right]_{j+1}^{T}=\tilde{M}_{j+1}^{-1}\left(\varrho_{j+1}\right) \cdot \tilde{M}_{j}\left(\rho_{j}\right) \cdot\left[\begin{array}{llll}
A & B & C & D
\end{array}\right]_{j}^{T}
$$

and $\tilde{M}_{j}$ is given by:

$$
\tilde{M}_{j}=\left(\begin{array}{cccc}
J\left(\gamma_{j} \rho\right) & Y\left(\gamma_{j} \rho\right) & 0 & 0 \\
\frac{n_{j}^{2}}{\gamma_{j}} J^{\prime}\left(\gamma_{j} \rho\right) & \frac{n_{j}^{2}}{\gamma_{j}} Y^{\prime}\left(\gamma_{j} \rho\right) & \frac{m \beta}{\rho \omega \varepsilon_{0} \gamma_{j}^{2}} J\left(\gamma_{j} \rho\right) & \frac{m \beta}{\rho \omega \varepsilon_{0} \gamma_{j}^{2}} Y\left(\gamma_{j} \rho\right) \\
0 & 0 & J\left(\gamma_{j} \rho\right) & Y\left(\gamma_{j} \rho\right) \\
\frac{m \beta}{\rho \omega \mu \gamma_{j}^{2}} J\left(\gamma_{j} \rho\right) & \frac{m \beta}{\rho \omega \mu \gamma_{j}^{2}} Y\left(\gamma_{j} \rho\right) & \frac{1}{\gamma_{j}} J^{\prime}\left(\gamma_{j} \rho\right) & \frac{1}{\gamma_{j}} Y^{\prime}\left(\gamma_{j} \rho\right)
\end{array}\right)
$$

where $\varepsilon$ and $\mu$ are the dielectric and magnetic susceptibilities, $\omega$ is the optical angular frequency, $\gamma_{j}=\sqrt{k_{j}^{2}-\beta^{2}}$, and the primes indicate derivative with respect to the function argument.

In the limit of strong vertical confinement (i.e., $\beta<<k_{j}$ ) it is possible to separate the modal field solutions into two distinct polarizations: TE consisting of $H_{z}, E_{\rho}$ and $E_{\theta}$ and TM consisting of $E_{z}, H_{\rho}$ and $H_{\theta}$. Each polarization component can be described by two coefficients in each layer: $A_{j}$ and $B_{j}$ for TM and $C_{j}$ and $D_{j}$ for TE. For each polarization, the boundary conditions at the interfaces between successive layers can be represented similarly to (4) using simplified $2 \mathrm{X} 2$ matrices:

$$
\tilde{M}_{j}^{T M}=\left(\begin{array}{cc}
J\left(\gamma_{j} \rho\right) & Y\left(\gamma_{j} \rho\right) \\
\frac{n_{j}^{2}}{\gamma_{j}} J^{\prime}\left(\gamma_{j} \rho\right) & \frac{n_{j}^{2}}{\gamma_{j}} Y^{\prime}\left(\gamma_{j} \rho\right)
\end{array}\right) \quad \tilde{M}_{j}^{T E}=\left(\begin{array}{cc}
J\left(\gamma_{j} \rho\right) & Y\left(\gamma_{j} \rho\right) \\
\frac{1}{\gamma_{j}} J^{\prime}\left(\gamma_{j} \rho\right) & \frac{1}{\gamma_{j}} Y^{\prime}\left(\gamma_{j} \rho\right)
\end{array}\right)
$$

Using relation (3) and the matrices (5), the field components can be "propagated" from the inner layers to the external layers. We use the finiteness of the field at $\rho=0$ so that $B_{1}=D_{1}=0$. The second boundary condition is no inward propagating field beyond the last layer, so that $B_{N+1}=-i A_{N+1}$ for TM and $C_{N+1}=-i D_{N+1}$ for TE, where $N$ is the number of layers.

\section{DESIGN RULES}

The transfer matrix formalism enables us to find the modal field distribution in the case of an arbitrary arrangement of annular concentric dielectric rings. However, we are especially interested in structures that can lead to a concentration of the modal energy near a predetermined radial distance, i.e. within the defect.

It has been shown that the best strategy to attain an exponential decrease (or increase) in the field intensity in the grating region is to position the interfaces of the layers at the zeros and extrema of the $z$ component of the field. ${ }^{12}$ The index profile and the field are calculated simultaneously, using the index to find the field and the field to determine the position of the interfaces. The resulting optimal index profile exhibits an inherent resemblance to the conventional (Cartesian) Bragg reflector. The optimal Cartesian Bragg reflector can be designed in a similar way, leading to layers that are quarter-wavelength thick. ${ }^{13}$ Here the resulting layers are also "quarter-wavelength" thick but in the sense of the quasiperiodicity of the $m^{\text {th }}$ order Bessel function. The defect (again, as in the Cartesian case) should be "half-wavelength" wide, i.e. its interfaces should be located at successive zeros of the field.

In order to attain a transverse field profile which is confined within the defect, the profile must exponentially increase for $\rho<\rho_{\text {def }}$, and exponentially decrease for $\rho>\rho_{\text {def. }}$. This requirement determines which index-interfaces (low $\rightarrow$ high or high $\rightarrow$ low) should be positioned at zeros of the field and which at the extrema of the field. The constraints on the index profile are similar to the Cartesian case and differ for the TE and TM polarizations. For the TE polarization, the 
interfaces for decreasing (increasing) field should be at the zeros (extrema) of $H_{z}$ if $n\left(\rho^{-}\right)>n\left(\rho^{+}\right)$at the interface and at the extrema (zeros) of $H_{z}$ if $n\left(\rho^{-}\right)<n\left(\rho^{+}\right)$at the interface. For the TM polarization the interfaces for decreasing (increasing) field should be at the extrema (zeros) of $E_{z}$ if $n\left(\rho^{-}\right)>n\left(\rho^{+}\right)$at the interface and at the zeros (extrema) of $E_{z}$ if $n\left(\rho^{-}\right)<n\left(\rho^{+}\right)$at the interface. The interfaces of the defect must be located at zeros of $H_{z}$ for TE and of $E_{z}$ for TM.

Figure 3 depicts the refractive index (Fig. 3(a)) and the TE modal field (Fig. 3(b)) profiles of an ABR designed for a $0.55 \mu \mathrm{m}$ thick InGaAsP layer suspended in air. The device is designed to have a mode with an angular propagation coefficient of $m=7$ at $\lambda_{\text {res }}=0.852 \mu \mathrm{m}$. The effective index approximation in the vertical dimension is used to reduce the 3D problem to a $2 \mathrm{D}$ equivalent one. As can be seen in the figure, the field is primarily confined in the defect and it decays while oscillating in the Bragg reflectors.
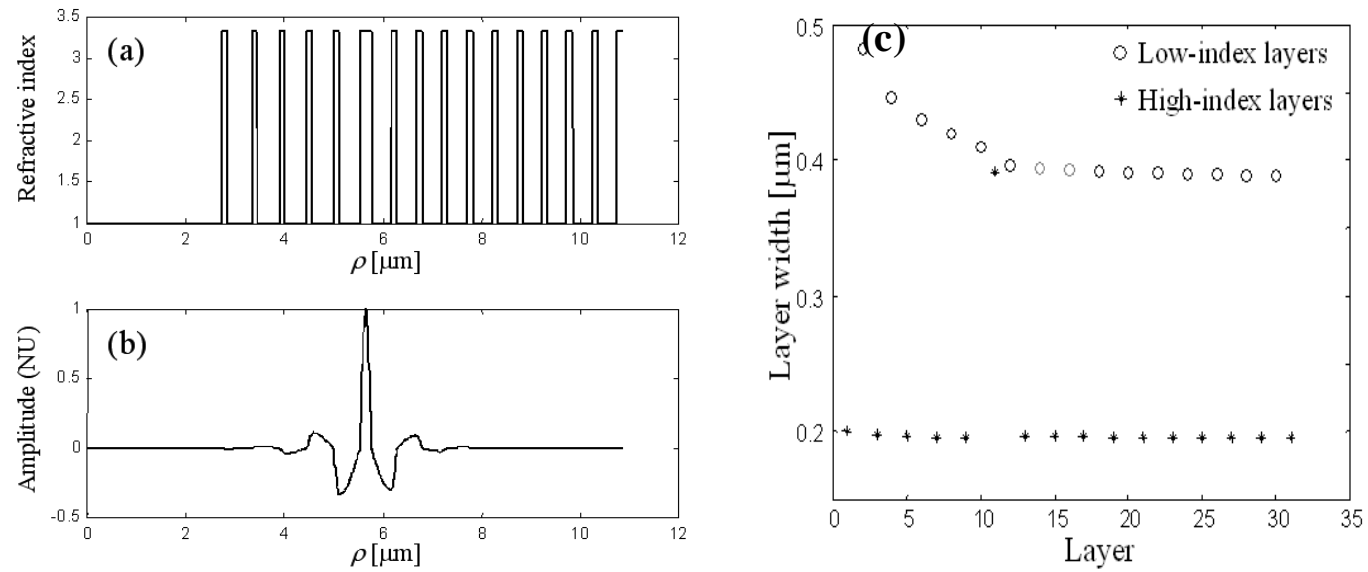

Figure 3. Refractive index (a) and TE-polarized modal field (b) profiles of an ABR designed for $m=7, \lambda_{\text {res }}=0.852 \mu \mathrm{m}$, with 5 internal and 10 external Bragg layers. (c) A non-periodic distribution of high- and low-index material is required in an annular Bragg resonator.

Figure 3c shows the widths of the Bragg layers as a function of the layer number of the ABR depicted in Fig. 3a. There are two notable properties of the Bragg layers: 1) the width of the high-index layers is smaller than the width of the lowindex layers and 2) the width of the layers decreases exponentially as a function of the radius, converging asymptotically to a constant value. The first property exists in conventional DBRs as well and stems from the dependence of the spatial oscillation period, or the "wavelength", on the index of refraction. The second property is unique to the cylindrical geometry and arises from the non-periodic nature of the solutions of the wave equation (Bessel or Hankel functions) in this geometry.

At large radii the widths of the Bragg layers converges asymptotically to the conventional (Cartesian) quarterwavelength condition. Mathematically, this can be explained by noting that for large radii the Bessel function can be approximated by a sinusoidal function divided by square root of the radius. From the physical point of view, at large radii the curvature of the Bragg layers is small and, therefore, the structure of a radial reflector structure at large radii is similar to the structure of a Cartesian reflector. Figure $3 \mathrm{~b}$ shows the radial mode profile of the ABR structure of Fig. $3 \mathrm{a}$. The field is confined within the defect and it decays while oscillating in the Bragg region. While the overall envelope of the radial modal profile (averaging over the oscillations) is quite similar to that of a conventional ring resonator, the oscillatory behavior of the field in the reflectors regions is the fingerprint of the distributed feedback mechanism.

\section{ADVANTAGES AND APPLICATIONS}

The Bragg confinement mechanism allows great flexibility in engineering the radial mode profile. For example, it is possible to design a resonator in which the light is confined within a defect composed of low refractive index material or 
even air. By contrast, this would be impossible in the case of conventional TIR-based resonators. Such configuration is useful for sensing applications as discussed below.

The Bragg reflection concept allows one to tailor the reflector structure to a desired radial field profile and decouple between the modal volume (or cavity dimensions) and the radiation losses. In fact, the radiation losses can be reduced almost arbitrarily, without significantly changing the modal volume, simply by adding more layers to the (external) Bragg reflector. Therefore, resonators exhibiting both high $Q$ and small modal volume can be engineered and their modal filed profile can be tailored for specific applications.

Another interesting and important property of the ABR structure is that it supports slow light propagation along the circumference of the defect. Intuitively, this property can be understood as follows: Because the Bragg reflector does not require the incidence angle of the EM wave to be larger than some "critical" angle (as in TIR based devices), it is possible to design an ABR in which the incidence angle of the wave is almost perpendicular to the Bragg layers. The angular component of the wave-vector in this case is very small, and thus, the angular propagation velocity is very low. This propagation mode has several advantages such as large gain (if the device is a laser), large and controllable dispersion and enhanced nonlinear response.

More specifically, the unique modal field profile and characteristics of the ABR structure are advantageous for biochemical sensing applications, surface emitting lasers and for studies involving strong atom-field interactions such as nonlinear optics and cavity QED.

\subsection{Sensing}

Among the most straightforward approaches for optoelectronic (bio)chemical sensing is to detect the change in the refractive index or the absorption caused by the presence of a (bio)chemical agent. Several schemes have been suggested to detect these types of changes, employing directional couplers, ${ }^{14}$ Mach-Zehnder interferometers (MZI) ${ }^{15}$ or high- $Q$ optical resonators. ${ }^{16}$ The detection mechanism underlying these sensors is the modification of the phase accumulation rate (i.e., the propagation coefficient) of the field due to the interaction of the evanescent tail of the field with the analyte.

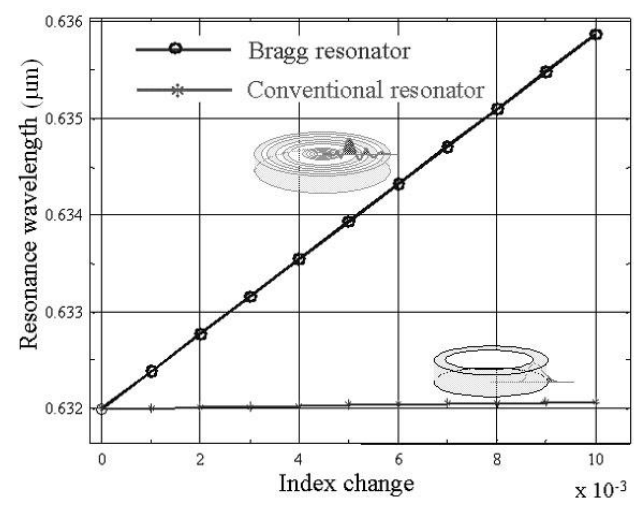

Figure 4. Comparison of the sensitivity of an $\mathrm{ABR}$ and a conventional ring resonator to changes in the refractive index of the surroundings.

The primary disadvantage of these detection methods is that the interaction of the field with the surrounding environment is weak, and therefore, the influence of the analyte on the propagation coefficient is small. As a result, achieving high sensitivity requires large interaction length leading to the requirement of long interferometers and very high- $Q$ resonators. In addition, the MZI-type sensors might have difficulties detecting small numbers (or single) molecules, regardless of their length. On the other hand, the ABR structure and mode profile (see Fig. 3) allow for the interaction of the non-evanescent part of the field, especially when the device is designed to include an air defect. As a result, ABR- 
based sensors are expected to offer significantly enhanced sensitivity compared to sensors that are based on conventional resonators of similar dimensions and materials.

Figure 4 shows a comparison between the shifts of the resonance frequency of an ABR and a conventional ring resonator due to changes in the refractive index of the surroundings. The ABR consists of alternating layers with refractive indices of 1.545 and 1.0 and an air defect. The conventional resonator consists of $n=1.545$ core surrounded by air cladding. Both resonators are approximately $16 \mu \mathrm{m}$ in diameter and designed to resonate in the visible wavelength regime. The sensitivity of each device is indicated by the slope of the curves shown in Fig. 4. The resonance wavelength of the conventional ring resonator shifts by approximately $0.007 \mathrm{~nm}$ for an increase of $10^{-3}$ in the refractive index. For the same index change, the Bragg resonator's resonance wavelength shifts by $0.4 \mathrm{~nm}$, i.e., the ABR exhibits higher sensitivity by a factor of 60 .

\subsection{Telecommunication Applications}

For telecommunications, low loss and small dimensions are desired for any resonator-based application. For example, the strong dispersion characteristics of the ABR can be exploited to realize a compact dispersion compensator or a compact laser source. Because of the low group velocity of the field in an ABR, the achievable intensity inside the cavity is much higher compared to conventional resonators with the same quality factor. This is because that for a given power level in the guiding channel, the corresponding field intensity is inversely proportional to the group velocity. Therefore, and ABR can exhibit a strong nonlinear response even for relatively modest input power levels and can be used to realize integrated all-optical processing elements. Moreover, the low group velocity (as well as the high $Q$ ) can be exploited to attain very low-threshold lasers. For such application, the ABR layer structure can be modified in order to efficiently emit light perpendicular to the surface (similar to VCSELs) by employing a second order Bragg reflection scheme - a Bragg reflector consisting of three-quarter wavelength layers. ${ }^{9}$ In such reflector, the first Fourier harmonic of the structure reflect the incidence wave in the vertical (out of plane) direction while the second Fourier harmonic is responsible for the in-plane reflection.

\subsection{Nonlinear Optics and Cavity QED}

Resonators exhibiting small modal volume and high $Q$ are excellent tools for studying the interaction of matter with strong electromagnetic fields. In such resonators, the intensity circulating in the cavity can be orders of magnitude stronger than the input intensity and it is concentrated in small volume. These characteristics of resonators have been exploited for demonstrating various nonlinear optical phenomena and entanglement photon-atom quantum states. ${ }^{7}$

In addition, to the high $Q$ and the small modal volume of the ABRs, the slow group velocity of the field circulating in the cavity even further. For a given power level in the guiding channel, the corresponding field intensity is inversely proportional to the group velocity. Consider, e.g., an ABR with a "wavelength" wide defect consisting of material with refractive index of 3.5 , which is design to resonate in the $m=8$ angular mode at $\lambda=1.55 \mu \mathrm{m}$. Using the formalism outlined in section 2, the group index of the device is evaluated and found to be $n_{\mathrm{g}}=c / V_{\mathrm{g}}=15.4$. Comparing to the maximal group index achievable in conventional resonators consisting of similar materials $\left(n_{\mathrm{g}} \sim 3.5\right)$, the discussed ABR's slower group velocity reduces the power level required for the observation of nonlinear optical phenomena by almost an order of magnitude.

Experiments in cavity QED require atom-photon interactions in the strong coupling regime. ${ }^{17}$ For this purpose, the cavity $Q$ must be as large as possible and the modal volume (i.e., field localization) as small as possible. PC defect cavities have drawn much attention as potential candidates for such experiments, offering both small modal volume of the order of cubic wavelength, and high $Q$. Recently, we demonstrated an ABR-like nanocavity with $Q \sim 2000$ and modal volume of 0.2 cubic wavelengths. ${ }^{11}$

By scaling the device dimensions, it is possible to shift the resonance wavelength of the nanocavity to $0.85 \mu \mathrm{m}$ which corresponds to the $\mathrm{D} 2$ atomic transition of ${ }^{133} \mathrm{Cs}$. The strong coupling regime is characterized by critical atom $\left(\mathrm{N}_{0}\right)$ and 
photon $\left(\mathrm{m}_{0}\right)$ that are smaller than one. ${ }^{17}$ Using the parameters of Ref. [18] for the Cesium atomic decay rate $\left(\gamma_{\perp}=2.6 \mathrm{MHz}\right)$, the critical atomic atom and photon numbers for the resonator shown in Fig. 2 are $\mathrm{N}_{0}=2.5 \times 10^{-3}$ and $\mathrm{m}_{0}=4.4 \times 10^{-8}$. These quantities are well below 1 , indicating that an atom embedded in the defect would interact strongly with the cavity field.

\section{DEVICE FABRICATION}

Annular Bragg resonators of several geometries and Bragg reflector orders were fabricated within a thin membrane of InGaAsP semiconductor material. ${ }^{19} \mathrm{~A}$ cross-section of the semiconductor epitaxial structure used is illustrated in figure 5. The layers were grown by MOCVD on a (100) oriented InP substrate. This structure consists of a 500nm thick InP buffer layer, followed by a $50 \mathrm{~nm} \operatorname{InGaAsP}\left(\lambda_{\mathrm{g}}=1.3 \mu \mathrm{m}\right)$ stop etch, and a $250 \mathrm{~nm} \mathrm{InP}$ sacrificial layer. A $250 \mathrm{~nm}$ membrane composed of $605 \AA$ InGaAsP $\left(\lambda_{\mathrm{g}}=1.1 \mu \mathrm{m}\right)$ layers sandwiching six $75 \AA$ quantum wells $(1 \%$ compressive strain) separated by $120 \AA$ InGaAsP barriers $\left(\lambda_{\mathrm{g}}=1.2 \mu \mathrm{m}, 0.5 \%\right.$ tensile strain $)$ completes the structure. Photoluminescence from the quantum wells peaks at a wavelength $\lambda=1559 \mathrm{~nm}$. Annular Bragg resonators were designed to have resonant wavelengths between $1.5-1.6 \mu \mathrm{m}$, for large overlap with the gain spectrum of the multi-quantum-well material.

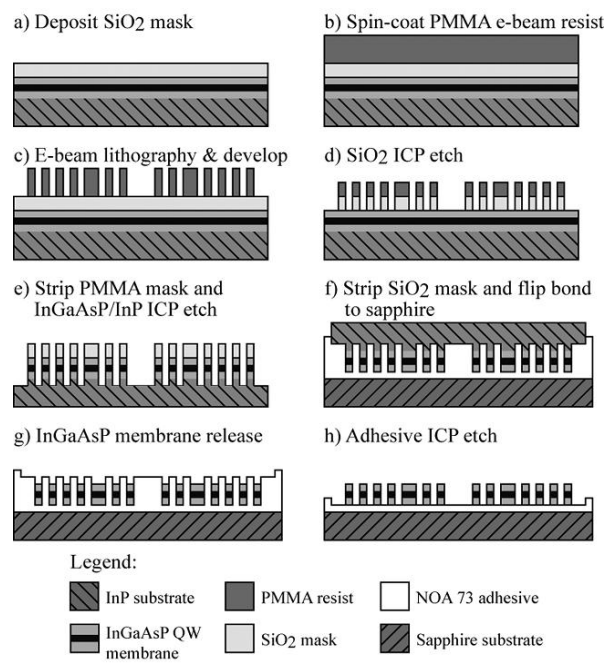

Figure 5. InGaAsP/InP multi-quantum-well semiconductor structure.

The optical gain of the semiconductor epitaxial structure used favors the TE polarization $\left(H_{z}\right)$, because of the optical properties of compressively strained quantum wells ${ }^{20}$. Therefore, the design of the fabricated devices is optimized for this polarization. In order to simplify the design calculations, we employ the effective index approximation in the vertical dimension. An effective index $n_{\text {eff }}=2.8$ is found by solving for the TE-polarized mode of the transferred InGaAsP slab. To facilitate the fabrication of the device, a mixed Bragg order scheme is used, with second-order $(3 \lambda / 4 \sim 430 \mathrm{~nm})$ highindex layers and first-order $(\lambda / 4 \sim 400 \mathrm{~nm})$ low-index layers.

In addition to relaxing the fabrication tolerances, the mixed Bragg order implementation induces a coherent diffraction component in the vertical direction. ${ }^{21}$ Although this mechanism reduces the overall $Q$ of the cavity, it facilitates the observation and measurement of the resonator emission.

The procedure followed to fabricate the annular Bragg resonators is as follows. First, an etch mask consisting of $120 \mathrm{~nm}$ of PECVD $\mathrm{SiO}_{2}$ was deposited on the semiconductor substrate. A $550 \mathrm{~nm}$ thick layer of PMMA electron-sensitive resist was spin-coated onto the substrate, and electron beam lithography was perfomed with a Leica EBPG 5000 direct-write system, to define the resonator patterns in the PMMA. The patterns were then transferred to the $\mathrm{SiO}_{2}$ layer with a 
reactive ion etching ( $\mathrm{RIE}$ ) step in $\mathrm{C}_{4} \mathrm{~F}_{8}$ plasma, after which the remaining PMMA was stripped using $\mathrm{O}_{2}$ plasma. A second RIE step was used to transfer the $\mathrm{SiO}_{2}$ pattern through the $250 \mathrm{~nm}$ thick InGaAsP membrane and approximately $75 \mathrm{~nm}$ into the InP sacrificial layer, using $\mathrm{HI} / \mathrm{H} 2 / \mathrm{Ar}$ plasma. Finally, the remaining $\mathrm{SiO}_{2}$ etch mask was removed with a buffered hydrofluoric acid wet chemical etch.

The high refractive index of the InP substrate $(n \approx 3.17$ at $\lambda=1.55 \mu \mathrm{m})$ reduces the vertical confinement of the guided optical mode within the membrane. Therefore, in order to achieve strong vertical optical confinement and improve the quality factor $\mathrm{Q}$ of the resonators, the patterned InGaAsP membrane must be surrounded by low index material. One method for achieving this is to generate an air-suspended membrane by selective removal of the InP beneath the device, as is commonly done with photonic crystal devices ${ }^{24}$. However, this technique is not applicable to the resonators studied here, because the concentric ring structure would collapse if the solid substrate were removed. Therefore, the resonators were flip-bonded to a double-side polished sapphire substrate with a thin layer of UV curable optical adhesive (Norland NOA $73, n \approx 1.54$ at $\lambda=1.55 \mu \mathrm{m}$ ). After fully curing the adhesive under a UV lamp, the InP substrate was removed using a combination of mechanical lapping and selective chemical etching. Note that the excellent thermal properties of sapphire can improve the conduction of heat generated during optical pumping away from the membrane. In addition, the large bandgap energy of sapphire, and associated transparency at visible and infrared wavelengths, permits optical pumping and imaging of the resonator luminescence from either the top or bottom of the device. Transfer of the semiconductor membrane to a transparent substrate also facilitates use of annular Bragg resonators for sensing applications. For instance, a solution containing an analyte whose concentration is to be monitored by the resonator could be introduced via the exposed topside of the device, while optical pumping of the resonator and imaging of the sensor signal could be accomplished through the transparent substrate.

SEM images of several completed devices are shown in Figure 6, prior to transfer of the InGaAsP membrane to the sapphire substrate. Images (A)-(C) show annular Bragg resonators with defect widths of $\lambda / 2 n$, and $3 \lambda / 2 n$. Odd-order defects were chosen to maximize modal overlap with the gain profile in the defect. A close examination of each reveals that the period of the Bragg gratings decreases moving radially outward from the center, as is required by the optimal design. The magnified view of the InGaAsP ridges in image (D) illustrates the very smooth vertical sidewalls achieved with our RIE process, which will minimize scattering of the strongly confined optical modes.
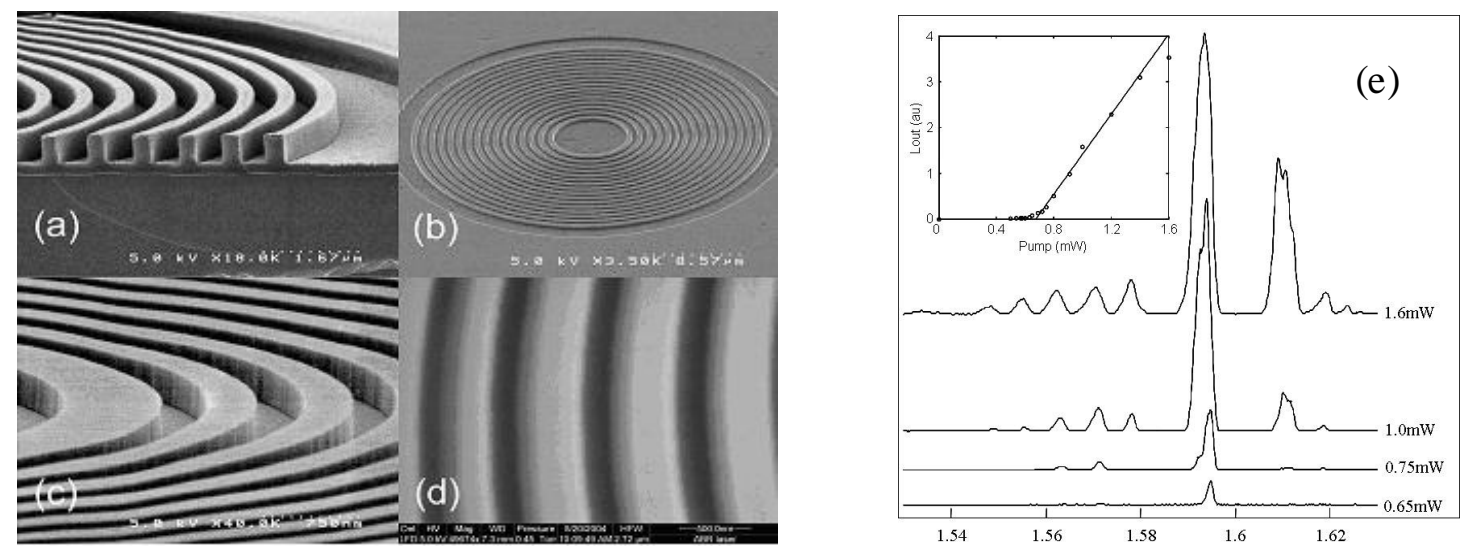

Figure 6. SEM images of completed annular Bragg resonators, taken before (a) and after RIE through the InGaAsP membrane. (a) A cross-section of an ABR with $\lambda / 2 n$ defect. (b) Top view. (c) $3 \lambda / 2 n$ defect. (d) Magnified image of $3 \lambda / 4 n$ wide InGaAsP ridges, illustrating smooth vertical sidewalls. (e) Optical spectra emitted from a lasing ABR under different pump levels. Inset: Integrated emitted power vs. pump power, showing laser threshold at $\sim 680 \mathrm{~mW}$. 


\section{MEASUREMENT RESULTS}

We measured and analyzed the vertical emission from the resonators under pulsed optical pumping. A Ti:sapphire modelocked laser was used to optically pump the devices at a center wavelength of $980 \mathrm{~nm}$, repetition rate of $76.6 \mathrm{MHz}$ and pulse duration of approximately $150 \mathrm{fs}$. A variable attenuator was used to control the pump power. The average pump power and center wavelength were monitored by a wavemeter, through a 50/50 beamsplitter. The pump beam is focused on the back side of the sample with a 50X objective lens. A $20 \mathrm{X}$ objective lens is used to collect the vertical emission from the sample and to focus it on an IR camera to obtain the NF intensity pattern and to couple the light into a multimode fiber to obtain the emitted spectrum. A CCD camera is used to image the resonators and the pump spot, for alignment purposes, using a white light source.

When the un-patterned QW layer structure is pumped, the emitted spectrum consists of a wide peak centered at $1559 \mathrm{~nm}$. As the pumping power is increased from $1 \mathrm{mw}$ to $20 \mathrm{~mW}$, the FWHM of the luminescence broadens from approximately $70 \mathrm{~nm}$ to $110 \mathrm{~nm}$, and the peak of the photoluminescence shifts towards longer wavelength due to heating. No significant shift is observed when the pump power is below $5 \mathrm{~mW}$, indicating that heating is of less significance at these pump levels. When an ABR is pumped, the emission characteristics change significantly. While the specific details (threshold levels, emitted wavelengths, etc.) vary from device to device, the overall behavior is similar. Once a certain pump intensity threshold is exceeded, clear and narrow ( 0.5nm FWHM) emission lines appear in the spectrum (see Fig. 6e). As the pump intensity is increased, the intensity of the emission lines increase as well and they broaden towards shorter wavelengths. Increasing the pump power further results in the appearance of additional emission lines.

To demonstrate the unique advantages of the ABR structure we focus on two applications - surface emitting lasers (SEL) and biochemical sensing.

\subsection{Surface emitting nanolasers}

Figure 7a depicts the emitted spectra from the $m=0$ laser for various pumping levels above the lasing threshold. An SEM image of the resonator is shown in Fig. $7 \mathrm{~b}$. The emitted light consists primarily of a single wavelength at $\lambda=1.56 \mu \mathrm{m}$, very close to the target design wavelength of $1.55 \mu \mathrm{m}$. The inset of Fig. 2 shows an L-L curve of the same device, indicating a threshold at $\mathrm{P}_{\mathrm{th}}=900 \mu \mathrm{W}$. It should be noted that the pump powers quoted indicate the overall power carried by the pump beam while the actual power absorbed by the QWs is significantly lower. At high pump levels $(\sim 1.5 \mathrm{xP}$ th $)$, two additional low-intensity emission lines appear at longer wavelengths $(\sim 1.59 \mu \mathrm{m})$ although the main emission line remained the dominant one. We attribute these modes to emission from the external Bragg grating region.

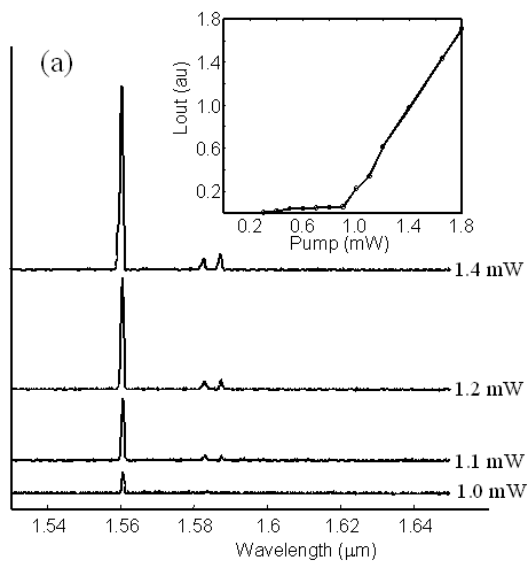

(b)

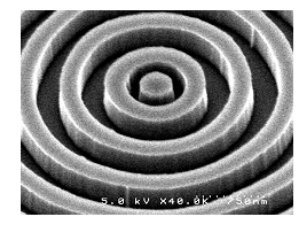

(c)

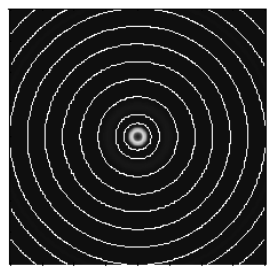

Figure 7. (a) The evolution of the emitted spectrum from the device shown in Fig. 1 as a function of the pump intensity. Inset - L-L curve, indicating a lasing threshold of $\mathrm{P}_{\mathrm{th}}=900 \mu \mathrm{W}$. (b) SEM image of the circular Bragg nanocavity designed to support the $m=0$ mode in the $300 \mathrm{~nm}$ wide central pillar. (c) Calculated modal intensity profile of the nanocavity 
Figure $7 \mathrm{c}$ shows a contour plot of the nanolaser index profile superimposed on a cross-section of the modal field intensity profile in the center of the active medium. As shown in the figure, the modal profile of the nanocavity is confined almost completely in the 300nm wide central pillar with a modal volume of $0.213(\lambda / n)^{3}\left(0.024 \mu \mathrm{m}^{3}\right)$ - only 1.75 times the theoretically possible limit of a cubic half wavelength. This modal volume is a $30 \%$ lower than the modal volume demonstrated in PC defect cavities ${ }^{22,23}$. This non-negligible improvement stems from the optimized match between the cavity dimensions and the (quasi) periodicity of the grating, made possible because of the cylindrically symmetric geometry.

The employment of second order radial Bragg gratings in the nanolaser provides, simultaneously, the radial feedback necessary for lasing and the vertical output coupling. Compared to conventional vertical cavity surface emitting lasers, the circular Bragg grating based SEL is simpler to fabricate and expected to provide higher output powers because of the larger field-gain overlap in the active material.

\subsection{Ultra-sensitive sensors}

Figures $8 \mathrm{a}$ and $8 \mathrm{~b}$ show respectively an SEM micrograph of an ABR and conventional ring based sensors realized within a thin membrane of InGaAsP active material. Figs. 8c and 8d depict a cross-section of the mode profile of the ABR and the conventional ring. The index structure is superimposed on the calculated mode profiles. The field profile of the ABR peaks in the defect and decays while oscillating in the reflector regions. Figs $8 \mathrm{c}$ and $8 \mathrm{~d}$ also illustrates the advantages of the ABR structure for sensing applications. Even though the main lobe of the mode profile does not interact with the device surroundings, significant part of the field within the trenches between the semiconductor rings are exposed to the surroundings, thus yielding large interaction volume (compared to a conventional resonator). Thus, an ABR based sensor is expected to exhibit enhanced sensitivity compared to a conventional resonator of similar dimensions.

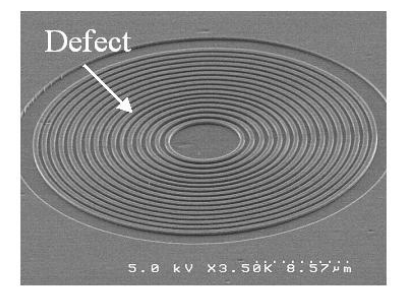

a

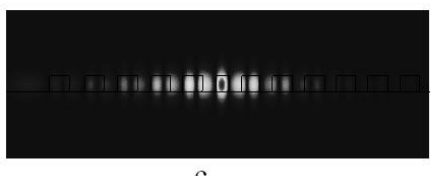

$\mathrm{c}$

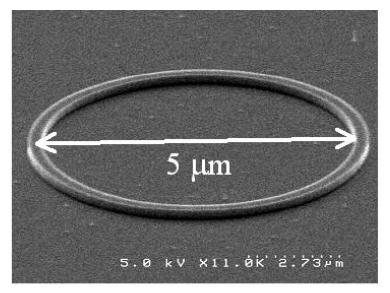

b

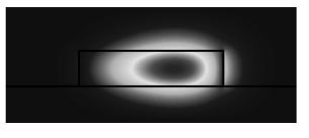

d

Figure 8. SEM images of the ABR (a) and the conventional ring (b) sensors, and their corresponding modal field profiles (c, d).

To test the influence of a change in the ambient refractive index on the laser spectrum, the ABR was immersed in a specially designed beaker containing index-matching fluids with different index of refraction. For each fluid, the emitted spectrum is measured $\left(P_{\text {pump }} \sim 2.5 \mathrm{~mW}\right.$

To demonstrate the advantages of ABR-sensors in terms of sensitivity and resolution, we compare the performance of our device to that of a conventional ring resonator with $5 \mu \mathrm{m}$ radius consisting of the same material. ${ }^{24}$ The conventional ring was tested under the same conditions (pump power, spot-size, etc.) that were used to characterize the ABR sensor. The sensitivity of the conventional resonator is approximately $d \lambda / d n \sim 33 \mathrm{~nm}$ (dashed) and the resonance FWHM is $\sim 1.4 \mathrm{~nm}$ wide, while the sensitivity of the ABR is $d \lambda / d n \sim 130 \mathrm{~nm}$ (solid) and the resonance FWHM is $\sim 1 \mathrm{~nm}$ wide. As a result, the advantage of the ABR as a sensor is two-fold: 1) The sensitivity of the ABR is 4 times larger than that of the 
conventional device because of the larger interaction volume and 2) the conventional resonator has wider linewidth because of its lower $Q$, which reduces the ability to resolve the resonance wavelength.

For the $1 \mathrm{~nm}$ linewidth of the ABR, shifts in the resonance wavelength of $0.1 \mathrm{~nm}$ can be easily resolved, thus allowing the detection of ambient index changes of $\Delta n \sim 5 \times 10^{-4}$, which to our knowledge is the one of the best resolutions ever demonstrated using integrated optical nano-sensors. ${ }^{25}$ As a comparison, for $1.4 \mathrm{~nm}$ FWHM of the conventional ring, the ability to resolve the resonance wavelength is limited to $\sim 0.14 \mathrm{~nm}$, which when combined with the lower sensitivity of the conventional device, yield a minimum detectable index change of $\Delta n \sim 4 \times 10^{-3}-$ approximately an order of magnitude less than that of the ABR sensor.

\section{CONCLUSIONS}

We have studied, experimentally and theoretically, the characteristics of a novel class of lasers that are based on radial Bragg reflectors. Lasing action with low threshold levels are demonstrated at room temperature under pulsed optical pumping. The observed $Q$ factors are between 1000 and 2000. The unique characteristics on these lasers make them promising candidates for numerous applications in telecommunications, sensing and basic research.

We demonstrated single mode lasing at telecommunications wavelengths from a circular Bragg surface emitting nanolaser with an ultra-small modal volume. In addition to their applicability for optical communication systems, such structures are highly attractive for studies involving strong atom-field interactions which require high optical intensities and small modal volumes.

Circular Bragg micro-cavities also offer significant enhancement of the ability to detect small changes in the ambient refractive index, compared to conventional micro-rings, because of their lager interaction volume and $Q$. We demonstrated, experimentally, sensitivity enhancement of an order of magnitude in an ABR compared to conventional resonators. Further enhancement of the ABR sensor sensitivity can be achieved by employing an air-defect design. The lasers' highly compact dimensions and enhanced resolution, make them excellent candidates for becoming key components for the realization of compact, integrated biochemical sensor arrays.

\section{ACKNOWLEDGMENTS}

This research was supported by the National Science Foundation and DARPA. 


\section{REFERENCES}

1. C. K. Madsen and J. H. Zhao, "Optical Filter Design and Analysis: A Signal Processing Approach", WileyInterscience Publications, New-York 1999, and references therein.

2. B. E. Little, Opt. Lett. 23, 1570 (1998)

3. J A. Yariv, IEEE Photonics Technol. Lett. 14, 483 (2002).

4. J. E. Heebner et al., J. Mod. Opt. 49, 2629 (2002).

5. C. Y. Chao et al., Appl. Phys. Lett. 83, 1527 (2003).

6. A. B. Matsko et al., J. Mod. Opt. 50, 2523 (2003).

7. See for example K.J. Vahala, Nature 424, 839-846 (2003) and references therein.

8. E. A. J. Marcatili, AT\&T Tech. J. 48, 2103 (1969).

9. J. Scheuer and A. Yariv, J. Opt. Soc. Am. B 20, 2285 (2003).

10. J. Scheuer, W. M. J. Green, G. DeRose and A. Yariv, Opt. Lett. 29, 2641 (2004).

11. J. Scheuer, W. M. J. Green, G. DeRose and A. Yariv, Appl. Phys. Lett. 86, 251101 (2005).

12. P. Yeh, A. Yariv and E. Marom, "Theory of Bragg fiber", J. Opt. Soc. Am. 68, 1196-1201, 1978.

13. See for example, A. Yariv, "Optical Electronics in Modern Communications" $5^{\text {th }}$ ed., Oxford University Press, NewYork, 1997.

14. B. J. Luff, R. D. Harris, J. S. Wilkinson, R. Wilson and D. J. Schiffrin, "Integrated-optical directional coupler biosensor," Opt. Lett. 21, pp. 618-620, 1996.

15. B. J. Luff, J. S. Wilkinson, J. Piehler, U. Hollenbach, J. Igenhoff and N. Fabricius, "Integrated optical MachZehnder biosensor," IEEE J. Lightwave Technol. 16, pp. 583-592, 1998.

16. R. E. Boyd and J. Heebner, "Sensitive Disk Resonator Photonic Biosensor," Appl. Opt. 40, pp. 5742-5747, 2001.

17. H.J. Kimble, in Cavity Quantum Electrodynamics, edited by P. Berman (Academic, San Diego, 1994).

18. J. Vučković, M. Lončar, H. Mabuchi, and A. Scherer, Phys. Rev. E. 65, 016608, 2001.

19. W. M. J. Green, J. Scheuer, G. DeRose, A. Yariv and A. Scherer, J. Vac. Sci. Technol. B. 22, p. 3206, 2004.

20. L. A. Coldren and S. W. Corzine, "Diode Lasers and Photonic Integrated Circuits", Wiley-Interscience Publications, New York, 1995.

21. S. Kim, H. Ryu, H. Park, G. Kim, Y. Choi, Y. Lee and J. Kim, "Two-dimensional photonic crystal hexagonal waveguide ring laser,” Appl. Phys. Lett., vol. 81, pp. 2499-2501, September 2002.

22. O. Painter, R. K. Lee, A. Scherer, A. Yariv, J. D. O'Brien, P. D. Dapkus and I. Kim, Science 284, 1819 (1999).

23. T. Yoshie, O. B. Shchekin, H. Chen, D. G. Deppe and A. Scherer, IEICE Trans. Electron. E87-C, 300 (2004).

24. W. M. J. Green, J. Scheuer, G. DeRose and A. Yariv, CLEO/QELS 2005, Baltimore, Maryland, paper QPDA7

25. E. Chow, A. Grot, L. W. Mirkarimi, M. Sigalas, and G. Girolami, "Ultracompact biochemical sensor built with twodimensional photonic crystal microcavity”, Opt. Lett. 29, 1093 (2004). 